\title{
GEBODEN EN REGELEN VAN DE LIEDEN VAN NAIPOSPOS
}

\author{
vermoedelijk geschreven door \\ GOEROE TINANDANGAN.
}

\author{
TWEEDE GEDEELTE (blz. 254 -280) \\ uit de nalatenschap van $H$. N. van der Tuuk \\ uitgegeven door P. Voorhoeve.
}

Het eerste gedeelte van deze adatbeschrijving van Naipospos is onlangs met vertaling en aantekeningen gepubliceerd door D. W. N. de Boer ${ }^{1}$ ). Van het tweede deel bestond reeds een vertaling door J. C. Vergouwen ${ }^{2}$ ). De tekst hiervan volgt hieronder, ontleend aan het Leidse handschrift Cod. Or. 3405 p. $205-280$, verder genoemd tekst A.

Over „Uhum”, d.i. adat, van boven-Baroes handelen ook de volgende teksten in het legaat Van der Tuuk:

B. Cod. 3406 p. 1-107 door Van der Tuuk, getiteld: Zeden, gewoonten en wetten van Pasaribu. Dit bestaat uit twee delen; het eerste, p. $1-56$, behandelt de plechtigheden die voor een mens plaats vinden van vóór zijn geboorte tot na de geboorte van zijn eerste kind. Het tweede gedeelte, p. 56-107, gaat over gebruiken in verband met de oorlog.

C. Cod. 3419 p. $121-131$, bevat na een kort opschrift, waarin als titel wordt genoemd „Uhum di Batak”, stukken over houtaankap, ontginning van bos, diefstal, overspel, weer over diefstal, moord; vervolgens enige algemene rechtsregels; daarna over marktstichting, en ten slotte over de geschenken bij het overlijden van een vrouw aan haar familie te geven. De bepalingen over diefstal zijn in de noten bij het hier volgende stuk van A vermeld.

1) In deze Bijdragen D1. 103 (1946), blz. 339-457. Verbeteringen in D1. 104 (1947), blz. 83-88.

2) Adatrechtbundel XXXV (1932), blz. 135-154, hier verder geciteerd als Vg. 
D. Cod. 3417 p. 105-106, door Van der Tuuk met Latijns karakter geschreven, getiteld Uhum, is een opsomming van boetes, die op diverse misdrijven staan. Al deze bepalingen zijn hieronder in noten genoemd.

E. Cod. 3403 p. 239 , eveneens in Latijns karakter, getiteld Uhum di Naipospos, is van dezelfde aard maar beknopter. Ook de inhoud hiervan is beneden in de noten medegedeeld.

F. Cod. 3406 p. 213-208, in Latijns karakter, Uhum di hami Batak, bevat eerst een stukje over de wijze van procederen; daarna over mangalangkup (het wegnemen van andermans vrouw; zie de noot bij $\mathrm{A}$ p. 254); over het uithuwelijken van een dochter; over huiselijke twisten.

G. Cod. 3401 p. $252-257$, in Latijns karakter, getiteld Uhum: enkele algemene rechtsregels; huwelijk; zwangerschap; geboorte en naar de rivier brengen van het kind; over de dood van de vrouw of het kind; over de oorlog.

Ten slotte bevat Cod. 3416 p. $238-288$ afschriften in Bataks karakter van stukken die voorkomen in $\mathrm{A}, \mathrm{C}$ en $\mathrm{G}$ en een stukje over algemene rechtsregels dat ontleend is aan het verhaal van Si Bobak (Cod. 3397 en elders, zie Voorhoeve, Overzicht no. 188; de inhoud van het bedoelde stuk vindt men daar p. 53-54 in het met B aangeduide gedeelte). Van p. 245 af is het een in regelmatig handschrift, blijkbaar door een djoeroetoelis gemaakt afschrift. Dezelfde hand heeft ook het voorafgaande stuk p. 225-238 geschreven. Hiertussen bevindt zich p. 238-244 een gedeelte, geschreven in een tamelijk stijve hand, misschien door Van der Tuuk zelf. Als titel heeft Van der Tuuk er bij geschreven: Fragmenten uit de wetgeving. Hier volgt een overzicht van de corresponderende stukken:

Cod. 3416 p. $238-241=$ (C) 3419 p. $121-126$ met veranderde volgorde.

p. 241-242 uit Si Bobak.

p. $242=(\mathrm{G}) 3401$ p. 252.

p. $242-244=$ (A) 3405 p. $254-256$.

p. $245-250=3416$ p. $238-244$.

p. $250-257=$ (A) 3405 p. $256-270$.

p. $258-261=($ C) 3419 p. $126-131$.

p. $262-286=$ (A) 3405 p. $205-254$.

p. $286-288=$ (A) 3405 p. $270-277$. 
In Cod. 3416 p. 249 heeft Van der Tuuk in de marge geschreven: vgl. No. I bl. 91 sqq. Hieruit blijkt, dat van dit gedeelte nog een, niet bewaarde, tekst bestaan moet hebben.

Voor het gedeelte, dat door Vergouwen en De Boer vertaald is, geeft A de beste tekst; het afschrift in Cod. 3416 heeft geen betere lezingen en kan dan ook verwaarloosd worden. In onderstaande tekstuitgave is dan ook uitsluitend Cod. 3405 gevolgd.

De cijfers in de marge geven de bladzijden van het handschrift aan; de tussen [ ] geplaatste cijfers in de tekst verwijzen naar Vergouwen's hoofdstuk- en paragraaf-indeling. De volgorde in de vertaling wijkt enigszins af van die in het handschrift. Hoofdst. 26-31, 7 vindt men op blz. $254-259$ van het handschrift; $31,8-31,17$ op blz. $259-261 ; 32,1$ blz. $259 ; 32,2$ blz. $262 ; 32,3$ blz. $259 ; 32,4-6$ blz. $261 ; 32,7$ blz. $265-266 ; 32,8$ blz. $266 ; 32$, 9 blz. $269 ; 32,10$ blz. 266 ; 32, 11-13 blz. 267-268; 32, 14 blz. 266; 32, 15 blz. 269270 ; 32, 16 blz. 266-267; 32, 17 blz. 269; 33-34 blz. 262-263; 35,1 blz. $270-273 ; 35,2-36$ blz. $276-280$.

Overbodige lettergrepen zijn tussen () geplaatst, toe te voegen lettergrepen tussen [ ].

254 [26] Ia uhum ni halak na mangalangkup tuan boru, molo didapot di ramba padua-dua dohot boru-boru i, molo dapot diihot, diboan ma tu huta. Diihot ma dohot boru-boru i. Hape molo so haihotansa, dibunu di ramba $i$, babi di eme ma goarna ${ }^{3}$ ). Tung adong pe donganna, na so djadi marlulu be i. Molo sai dimusui nampunasa djolma $i$, dialo ma, sai na talu do parlulu i. Alai pardjolma pe na so djadi do mandok be tu dongan ni na binununa, ianggo tu parboru sai na mangido uras ma nampunasa djolma. Hape molo na asi roha ni parboru i mida boruna tu tali dohot tu beangan, dilehon ma mas sabinsang ${ }^{4}$ ), djuhut sada ${ }^{5}$ ), dahanon saparmasan. Molo nungnga dilehon parboru $\mathrm{i}$, na so djadi be tu beangan boru $\mathrm{i}$. $\mathrm{Ba}$, ia na sanga dihoti do lahilahi i, sai ${ }^{6}$ ) boru-boru i ma padiri napuran panganonna dohot timbahona. Ia tung adong do na mangambati, dihata ma utangna: 12

3) goarna; zo in het hs. veranderd i.p. van $i$, maar later weer doorgestreept.

4) Vg., wel op grond van Batakse inlichtingen: een binsang goud $=20$ Spaanse matten). In deze tekst blijkt echter niets van een verschil tussen mas sabinsang en sabinsang; de binsang, oorspronkelijk een goudgewicht, stond in die tijd blijkbaar gelijk met 4 realen.

ऽ) Vg. steeds: een deel vlees; lees: een slachtbeest.

6) Vg. en blijft; lees: dan blijft.

D1. 105. 
binsang ${ }^{7}$ ), 1 horbo panulana ${ }^{8}$ ), 12 sibalambung dahanon. Ia dung dipangan na mandjalo i panula $i$, didjalo dohot dangdang $i$, asa mangalehon muse dape suhut na mardangdang $\mathrm{i}^{9}$ ) sabinsang pudun ni pangulu, dilehon págo-pago 2 riar tu radja na torop, dilehon partuptupan sariar tu namora ni huta. Hape molo soada na mangambati 255 donganna, dipangan ma na mangalangkup / i. Molo tole ma laho marboan tu harungguan mangebangkon tu radja ni ladang, asa dibarishon ma $\mathrm{i}^{10}$ ). Molo dung ro di harungguan i, ditalutukkon ma i. Dung ni asa marhata ma nasida di harungguan i. Dung sun marhata, mulak be ma nasida sian harungguan i. Dung ni diboan ma tu huta na niihotanna $i^{11}$ ). Dung ni, molo nadong dongan ni na tu talutuk $i$, asa dipangido nampunasa djolma i ma sira dohot asom dohot holat dohot sosar dohot panduduran. Ia godang ni panduduran 2 binsang, 1 djuhut, sadampang dahanonna. Ia godang ni asom dohot sira dohot holat dohot sosar sabinsang, 1 djuhut, saparmasan dahanonna. Ia dung didjalo i, panganonna ma djolma pangalangkup i. Ia uhum ni halak na mangan djolma,

7) Volgens E, eveneens uit Naipospos, is de boete voor een pangalangkup 30 binsang. Volgens D: 33 binsang, een buffel ter waarde van 8 realen, 3 ampang rijst, 1 reaal als pangolting (voor het vastbinden van de rekenstokjes), 4 realen als pago-pago (bezegelingsgeld) voor de bemiddelaar, 1 reaal als lapik $n i$ isang-isang (onderlaag voor de kinnebak van de karbouw; vgl. de term lapik sulang in Dairi, Ypes p. 104), 1/2 reaal als palu-palu ni gordang (lett. slagstok van de trom), 1 reaal als rompu pustaha (rotanband voor het wichelboek). Volgens F: 33 binsang en als panula een buffel die is balga binalgaan mokmok minokmohan, d.w.z. een eerste klas forse en vette karbouw. Is zijn snuit niet fors genoeg, dan moet daarvoor een binsang bijbetaald worden, evenzo als zijn oren te smal zijn, voor iedere poot die te dun mocht zijn en voor de staart als die te kort is. - Bij de hierboven gegeven verklaring van pangolting is aangenomen dat het staat voor pangolting rudji. Van der Tuuk merkt in zijn spraakkunst p. $180 \mathrm{op}$, dat de vorm onregelmatig is, daar het werkwoord luidt mangkolting. In Angkola is dit echter mangolting (volgens het woordenboek van Eggink); de adatbetaling heet daar, volgens Daulay in TBG 81 p. 574, holting rudji. Vergouwen noemt in Het Rechtsleven der Toba-Bataks de termen mangkolting en pangkolting bij het ontbinden van een huwelijk. In onze teksten heeft het een zeer ruime betekenis, daar het niet alleen in verband met de bruidschat maar ook bij andere betalingen (boete op diefstal enz.) voorkomt.

8) panula, vdT.: het bedrag van vlees, of wel een maal, dat de verliezende partij nog boven de boete (dangdang) aan de overwinnende te betalen heeft; welk maal de beide partijen met de rechtsprekenden gewoon zijn te delen. Vg.'s vertaling past niet op andere plaatsen waar het woord voorkomt.

$\left.{ }^{9}\right)$ d.i. de beboete; deze heeft boven zijn boete nog de pudun ni pangulu, pago-pago en partuptupan genoemde bedragen te betalen, die door $\mathrm{Vg}$. ten onrechte ten laste van de beledigde echtgenoot gebracht worden.

10) Vert.: Als men hem naar de vergadering gaat brengen om hem aan de hoofden van het landschap te vertonen, dan doet men dat onder het lossen van geweerschoten.

11) Vert.: Daarna wordt de gebondene naar het dorp (terug)gebracht. 
sintap dua borngin nari asa panganonna, indadong be mangan indahan i dibahen, tombal nitak na ma dibahen panganonna ${ }^{12}$ ). Ia tole mamanganhon, asa diboan ma tu balian, didokkon ma na mangalapi radja di na saharungguan i. Dung ro di balian, asa ditallik ma anak ni haju tolu, dung ni dipagohon ma i, dibahen ma pinardalihan. Dung ni ditalutukkon ma djolma i. Dung ni dihaliangkon ma mare-mare tu talutukna i. Dung ni digondang-gondangi ma i. Dung ni dibahen ma idjuk bulang-bulangna ${ }^{13}$ ). Dung ni dibuat suhut i ma babi sada bahen sosarna ${ }^{14}$ ), i ma djolo tambul ni radja na ro i dohot pola diinum. Dung ni dibuat ma parbue ni rube, dihaliangkon ma di lambung ni na di talutuk i. Dung ni ro ma radja nialapna i saluhut. Dung ni dilehon ma djuhut inon dohot pola tu radja na ro $i$. Dung sun nasida minum, marhata ma nasida. Sun marhata, asa dihatahon suhut i ma santi-santi dohot sitompion. Dung sun dihatahon, didaik ma sitompion i, dibahen tu bogasan angkadangan. Dung ni didaik ma dohot ogung dohot gordang ${ }^{15}$ ). Dung ni ditullang ma djolma na di talutuk i. Dung ni diparsahe-sahe 256 ma tutu. Dung sun dibagi-bagi halak, laho be ma, mula/k be ma halak tu hutana. Nasa na so sanga mulak, tu huta ni na mangalap $\mathrm{i}$ ma marborngin, Dung sun i, torang ni arina dipasang ma harungguan. Dung ni manungkun ma namora di hata ni harungguan. Dung ni dipaboa ma tutu. Dung ni diuhumi ma nasida dohot hula-hulana i. Dung ni dibahen ma utang ni hula-hulana i 2 binsang, 1 djuhut, sasibalambung [dahanonna]; daon ni latong ma i dohot daon ni tahuru dohot tobus ni obuk dohot pinggol. Didjalo ma i sian hula-hulana i. Dung ni diparhadjimot ma djolmana. Hape molo soada hula-hula mangalehon i, molo na gadisonna boru-boru i, digadis ma tutu; na so djadi be mandok hula-hulana. Tole laho margadis digotap ma udjung ni obukna $i$ asa adong pandangna diida halak. I ma uhum ni. Barang na dapot di bagas pe ibana, molo na dung djolma ni halak dipardjolma, molo soada na mangambati, sai panganon ma i.

[27] Ia uhum ni halak na manggombangi na hasae-saean, molo tardjumpa di halak, barang boru na manorlahon, sibeangkononhon ma i.

12) Vert.: Van twee dagen, voordat men hem opeet, geeft men hem geen rijst meer te eten, doch slechts meel(koeken).

13) De tekst zegt niet, dat de hoofddoek van idjoek ,voor het slachtoffer" gemaakt wordt; ik vermoed eerder: voor de slachtpaal.

14) Hier blijkt, dat van het door de verwanten onder de naam van sosar betaalde bedrag een varken wordt bekostigd om de uitgenodigde hoofden te onthalen.

15) Deze zin ontbreekt bij Vg.. Vert.: Daarop bergt men ook de bekkens en de pauken op. (De executie vindt dus niet onder opzwepend gondang-spel maar in een doodse stilte plaats). 
Indadong djadi i tu talutuk bahenon, na so djadi do i panganon, utangna do sihataon. Ia ma godang ni utangna, molo na saut di ibana boruboru i, $9^{16}$ ) binsang utangna, 1 djuhut parbinsangi, 3 parmasan dahanonna; i ma utangna ${ }^{17}$ ). Hape molo soada saut di ibana boru-boru $i$, 3 binsang utangna, 1 djuhut parbaribaan, sadampang dahanonna; i ma utangna. Hape molo na soada tondongna mangambati barang donganna, tu paradopan ma i. Hape molo soada na maniop mangadop, gadison ma i. Alai molo margadis ma, di lahi-lahi parabitan ni boru-boru i ma lahi-lahi $\mathrm{i}^{18}$ ), atupanna ma napuran, pulosonna pangisapan, dilehon tu na targadis $i$, diandungi ma na laho tu pargadisan . . . ${ }^{19}$ ).

[28] Ia uhum ni halak na mambahen rohana lahi-lahi tu boru-boru 257 na dipardongan di ramba, molo adong tanda ni lahi-lahi ditiop b/oruboru, laho pe lahi-lahi barang tu dia, sai na paimaonna do i. Hape molo na dung djolma ni halak boru-boru i, dilehon ma tanda tiniopna $i$ tu amana barang tu inana. Dung ni molo na so dapot disoro do lahi-lahi $i$, tu radja ma dohot tu namora dipaboa. Dung ni diuhumi ma i, marutang ma dibahen. Ia godang ni utangna: 7 binsang, 1 babi na bolon, 3 sibalambung dahanonna. Ia boru-boru $i$, indadong saut be di ibana. Hape molo na so djolma ni halak dape boru-boru i, didjadjang amana ma boruna i laho mahiompo. Dung ni asa laho ma boruna $i$ tu bagas ni paranak i. Dung ni ro ma hula-hula ni boru i, diihuthon ma boruna i, dironron ma paranak i. Dung ni marhata ma radja dohot namora. Dung ni dihata ma utang ni paranak i. Molo haidjuran ma, dilehon 4 binsang, 1 babi na bolon, 2 sibalambung dahanonna, i ma haidjuran. Molo nungnga dilehon paranak i, mulak ma parboru i, mulak ma dohot boruna i. Hape molo tu hata saut ma ninna paranak, saut di anakna ma boru i, dibuat ma djuhut sada, dilehon mas 2 binsang, daon ni sala ma i. Dung pe i asa dihata parhulaon, utang pangolion ma digarar. I ma uhum ni halak na tumurut rohana.

16) Zo in de tekst; Vg. één binsang.

17) Volgens E: 12 binsang, een volwassen varken en 1 binsang als pudion $n i$ pangulu (teken voor de bemiddelaar). In C en D wordt onder gombang verstaan overspel met een gehuwde vrouw. Volgens D bedraagt de boete slechts: $11 / 2$ binsang plus 1 binsang als kleed voor de man, 1 binsang als kleed voor de vader, voor de moeder $1 / 2$ binsang, voor de marga-genoten 1 reaal, voor de anakboru 1 reaal een varken ter waarde van 4 realen, 3 paromasan (=1 $1 / 2$ ampang) rijst en 2 realen voor si patei hata (die de zaak tot een einde brengt). C behandelt dit geval op dezelfde wijze als dat van een pangalangkup bij $\mathrm{A}$; de boete bedraagt 30 of 33 binsang.

18) De tekst is hier niet in orde; wellicht moeten de woorden di lahi-lahi wegvallen. Vg.'s vertaling zal wel juist zijn.

19) Herhaling van het opschrift aan het begin van het hoofdstuk. 
[29] Ia uhum ni halak na mangabing boru sian ladang barang sian pargadongan, dung diboan tu hutana torang ni arina marhara ma parboru $i$ tu huta na pulik. Dung ni laho ma nasida tu huta ni paranak i. Dung ni manomba ma pangabing $i$, dibuat ma djuhut panganon ni parboru i. Torang ni arina dibuat djuhut sada, dilehon mas 5 binsang daon ni na hodjor. Dung pe i asa dihata utang pangolion. Manodo ma parboru i di mas sidjaloonna. Dung ni mulak ma nasida tu hutana .... [30] Ia uhum ni halak na paulak djolma tu hula-hulana, molo na sumaburhon gambirna boru-boru barang na tardum dahanon di losung dibahen na mulak, gurgur ma pansamotan paulahon ni parboru. Hape 258 molo na soada do dosa ni boru-boru, tung di/paulak djolma, barang na mahilolong ibana sian djolmana dibahen dipaulak tu hula-hulana, tanduk ni mas indadong i sigararon ni parboru, djuhut hata saut indadong gararon ni parboru, dohot na muhut indadong gararonna i. Molo nungnga dilehon parboru $i$, asa dilehon boru i ma lage sada hadjut sada tu na paulakkon i. Dung ni mulak ma i tu hutana. Molo adong halak na siol, dibuat halak ma boru inon . . . ${ }^{20}$ ).

[31, 1] Ia uhum ni utang tangko, molo sitangko horbo 10 binsang, 1 babi na bolon, 3 ampang dahanonna. Hape molo na soada gararna barang na soada donganna mangambati, lomo ni roha ni suhut nama disi ; ia na tu gadis dibahen, djadi do; ia dipangan pe, djadi do $\ldots{ }^{21}$ ).

20) Op het huwelijksrecht hebben nog betrekking de volgende bepalingen: D. De schuld van sipahuempe, d.i. in geval een meisje toeloopt naar de man van haar keuze. Volgens Patik dohot Uhum p. 4 (vertaling Vergouwen in Adatrechtbundel XXXV p. 5) is de hoegrootheid van de bruidschat in dit geval geheel afhankelijk van de jonkman. D echter geeft het volgende tarief : 13 rudji (bestanddelen van de bruidschat), een varken van 2 realen met bijbehorend geldbedrag van 4 realen, gekookte rijst zoveel als de gasten op kunnen en een reaal voor de scheidsrechter (si tolai hata). - E. In geval van kinderloosheid kan de echtgenoot zijn bruidschat terug eisen en de bij het huwelijk geslachte dieren vergoed krijgen; hij heeft echter de ontvangen ulos (tegengeschenk van de vrouwsfamilie) terug te geven. - De stukken over het sluiten van een huwelijk, die in $\mathrm{F}$ en $\mathrm{G}$ voorkomen, kunnen het best bij een uitgave van $\mathrm{B}$ behandeld worden.

21) Boete voor een karbouwendief: D: 1 binsang als kleed (abit) voor de eigenaar, 3 ampang rijst, $1 / 2$ binsang als herinneringsgeld (ingot-ingot) voor de oudsten, 1 reaal als pangolting. C: vergoeding van de waarde plus 2 ampang rijst als pasuang harihir (herstel van de neusring). Van der Tuuk geeft voor pasuang harihir: de boete, die een buffel-dief betalen moet. Uit A p. 266 ivertaling p. 144) blijkt echter, dat met deze en soortgelijke termen in de eerste plaats de vergoeding van de waarde van het dier bedoeld wordt, waarbij dan nog een boete komt. - E geeft als algemene boete voor (grote) diefstallen op: 6 binsang en een volwassen varken; kan de dief dit niet betalen, dan wordt hij verkocht. Wordt de zaak voor de grote vergadering (hamungguan na godang) gebracht, dan moet 1 reaal betaald worden voor de kosten (pinangan ni harungguan), waarna men zich bij de beslissing van de radja's moet neerleggen. 
[31, 2] Ia uhum ni sitangko lombu, 9 binsang godang ni utangna, 1 babi na bolon, $2^{22}$ ) ampang dahanonna. Hape molo na soada donganna mangambati, songon sitangko horbo $i$ do bahenon ni suhut $i$... . [31,3] Ia godang ni utang ni sitangko babi, 3 binsang, 1 babi parbaribaan ${ }^{23}$ ), tolu parmasan dahanonna. Molo soada gararna barang donganna soada mangambati, djolma mangadop ma i, barang tu parsingir i pe mangadop, barang tu na pulik pe mangadop; anggo gadison indadong djadi . . . ${ }^{24}$ ).

[31, 4] Ia godang ni utang ni sitangko biang, sabinsang, 1 babi parpitu-hupangi, sasibalambung dahanonna. Molo na soada gararna, djolma mangadop ma $\mathrm{i}$; anggo gadison indadong djadi $\ldots{ }^{25}$ ).

[31, 5] Ia godang ni utang ni sitangko manuk, padua lombu ${ }^{26}$ ), 1 djuhut parbaribaan, sasibalambung dahanonna. Molo na soada donganna mangambati, djolma mangadop do $\mathrm{i}$; anggo tu gadis indadong djadi . . . ${ }^{27}$ ).

[31, 6] Ia utang ni sitangko eme sian sopo, 1 babi na bolon; molo na sian hauma ditangko barang eme sigotilon ditangko, 1 babi na 259 bolon, sabinsang batuna ${ }^{28}$ ), sa/dampang dahanonna $\left.\ldots . .{ }^{29}\right)$.

[31, 7] Ia godang ni utang ni sitangko mas, $12^{30}$ ) binsang, 1 horbo panulana, 12 sibalambung dahanonna. Hape molo soada na manggarar,

22) 2 , niet 3 .

23) Vg. vertaalt parbaribaan hier met halfwas; juister wschl. als in hoofdstuk 27: ter waarde van $1 / 2$ binsang.

24) Boete voor een varkensdief: E eveneens 3 binsang en het bijbehorende slachtdier. D : pasuang palangka (herstel van de trog) heet de boete, bestaande in een varken om op te eten, een levend varken en een hupang als herinneringsgeld voor de oudsten. C: eveneens pasuang palangka, bestaande in vergoeding voor het varken, 1 reaal en 1 ampang rijst.

25) Boete voor een hondedief: D: pasuang ungur-ungur (herstel van de hondehalsband met bamboe). C: pasuang ungur-ungur bestaande in vergoeding van de waarde van de hond, uang satali en 1 djual (= $1 / 4$ ampang) rijst. Verder vermeldt $\mathrm{C}$ dat de boete voor het stelen van een geit is pasuang hulanghulang (herstel van de halsband), d.i. de waarde plus 1 reaal en 1 ampang rijst. $\mathrm{Hij}$, die een dier steelt, dat in de strik van een ander gevangen is, heeft twee maal de waarde te vergoeden plus 1 reaal en 1 ampang rijst.

26) padua lombu $=6$ riar (dus $11 / 2$ binsang); vdT wdb. blz. $549 . \mathrm{Er}$ is dus geen sprake van een kip plus een koe.

${ }^{27}$ ) Boete voor een kippendief: D: $1 \frac{1}{2}$ binsang, een varken ter waarde van $1 / 4$ binsang, 3 djual rijst, $1 / 2$ reaal als herinneringsgeld voor de oudsten. E: 2 binsang plus een slachtbeest. C: $11 / 2$ binsang; geen vergaderkosten (na so nialap ni longit); een slachtbeest van $1 / 2$ binsang en 3 djual rijst.

28) batu is een bedrag in geld behorende bij een boete in vlees; het omgekeerde dus van pamula, een slachtbeest behorende bij een boete in geld.

29) Boete op diefstal van padi: E: $11 / 2$ binsang.

30) 12 , niet 21 ; 12 binsang is de maximum boete. 
sipanganon ma i. Adong pe gararna, molo soada lomo roha ni suhut, molo naeng panganonna di rohana, sai dipangan do i.... ${ }^{31}$ ).

$[32,1]$ Ia uhum ni halak na mamunu djolma, na so tandap binoto parbadaan, mata dipolsap do i mata pinolsap, djolma dibunu djolma binunu, molo dapot na mamunu i. Hape molo so dapot, anggo tu inana do sidohonon barang tu amana barang tu tondongna, indadong djadi $\mathrm{i}$ bunuon, utangna do sihataon. Ia godang ni utangna: 12 binsang, 1 horbo panulana, 12 sibalambung dahanon. Hape molo so targararsa, $\mathrm{i}[\mathrm{a}]$ adong halak na maniop, mangadop ma i. Hape molo soada halak na maniop mangadop, lomo ni roha ni parsingir nama disi; ia na gadisonna di rohana, digadis ma parutang $\mathrm{i}$; molo naeng hatobanna di rohana, diparhatoban ma i. Indadong be lungun roha ni halak di parsingir.... ${ }^{32}$ ).

$[32,3]$ Ia uhum ni halak na mamangus djolma, sipanganon ma i molo dapot. Hape ianggo tu donganna do sidohonon, utangna do sihataon. Ia godang ni utangna: 10 binsang, 1 horbo panulana, 10 sibalambung dahanonna. Hape molo soada targararsa barang soada donganna mangambati, songon sibunu djolma do i dibahen....

$[31,8]$ Ia uhum ni halak na mangaranda napuran, molo dapot sinoro, ihotan do i. Dung pe i asa hinata utangna. Ia godangna: 1 djuhut parriari, 2 riar hepeng, saparmasan dahannonna. Ia soada gararna barang tondongna na manggarar, diparutangkon djolo, molo adong na manahani. Hape molo soada na manahani, diadop utangna i, barang 260 tu na pulik/pe djadi do $\ldots{ }^{33}$ ).

Ia utang ni sitangko gadong, ia dapot sinoro, ihotan ma i. Dung pe i asa hinata utangna. Ia godang ni utang: 1 biang na bolon, sasuhat dahanonna, sariar hepeng $\left.\ldots .{ }^{34}\right)$.

31) Boete op diefstal van goud (d.w.z. gouden sieraden) volgens D : 1 buffel, 3 ampang rijst, 1 reaal als pangolting, 5 binsang als bijkomend bedrag in geld (batu-batu), $1 / 2$ binsang als bezegelingsgeld aan de oudsten.

32) Een moordenaar (pandobo si bum djolma) moet volgens D betalen: 2 binsang als lijkwade (saput), 5 binsang als weergeld (abul), 1 buffel, 1 binsang als rouwkleed (tudjung-tudjung) voor de overlevenden, 3 ampang rijst, 1 binsang als herinneringsgeld voor de bemiddelaar, $1 / 2$ binsang als pangolting; hij moet een maaltijd aanbieden aan (disauthon) de naaste schoonfamilie (parbonaan) en de schoonzoonsfamilie (amakboru) van den overledene. - Even groot is de boete voor een verrader (pardjehe-djehe) en een mensenrover (pamangus). Een gifmenger (parrasun) heeft te betalen 20 binsang, 1 buffel, 3 ampang .rijst, $1 / 2$ binsang als pangolting, 1 binsang als bezegelingsgeld voor de bemiddelaar. - C noemt ten dele weer andere betalingen voor een moordenaar (pandobo).

33) Boete voor een sirih-dief: E: 3 ringgit. D: 1 reaal. C: een slachtbeest van $1 / 2$ binsang en 1 binsang goud.

34) Deze paragraaf ontbreekt in de vertaling: Wie oebi steelt, mag, als hij met zijn buit gepakt wordt, gebonden worden; eerst daarna wordt de straf 
[31, 9] Ia utang ni sitangko djagung, $\mathrm{m}[\mathrm{olo}]$ dapot sinoro, ihotan do i. Dung pe i asa hinata utangna. Ia godang ni utangna: 1 biang na bolon, sasuhat dahanonna.... ${ }^{35}$ ).

$[31,10]$ Ia utang ni sitangko tobu harean $\left.{ }^{36}\right): 1$ manuk sabungan, 2 solup dahanonna....

[31, 11a] Ia utang ni sibuat tuak djantar ${ }^{37}$ ) : 1 babi parriari, sadjual dahanonna....

[31, 11b] Ia utang ni sitarus pola borngin, molo tarsoro, siihotan ma i. Dung pe i asa hinata utangna. Ia godang ni utangna: 1 babi parriari, sadjual dahanon, sariar hepeng.... $\left.{ }^{38}\right)$.

$[31,12]$ Ia uhum ni halak na manangko suhat lompisan ${ }^{39}$ ), molo dapot sinoro, ihotan ma i. Dung pe i asa hinata utangna. Ia godang ni utangna: manuk lahi bini ${ }^{40}$ ), dahanon sasuhat....

[31, 13a] Ia utang ni sitangko bubu: 1 manuk, dahanon 2 solup $\left.\ldots{ }^{41}\right)$. $[31,13 \mathrm{~b}]$ Ia utang ni sitangko tanggal ${ }^{42}$ ) : 1 biang na bolon, sasuhat dahanonna $\left.\ldots{ }^{43}\right)$.

[31, 14] Ia utang ni sitangko dahanon: 1 manuk ina-ina, 2 solup dahanonna. Alai molo dahanon asean tinangkona: 1 babi parriari, sadjual dahanonna....

$[31,15]$ Ia utang ni halak sitangko indahan sian gasingan, molo dapot disoro, diihoti ma i. Dung pe $i$ asa hinata utangna. Ia godang ni utang: 1 babi parriari, sadjual dahanonna. Molo soada olo panangko i mang-

garar, tu harungguan ma diboanhon. Ala/i molo sanga tu harungguan

behandeld. De straf is een volwassen hond, een soehat rijst en een reaal van 320 duiten.

Bij $\mathrm{E}$ bedraagt de boete voor een oebi-dief : 1 ringgit.

35) Boete voor een mais-dief: C: een slachtbeest van $1 / 4$ binsang en $1 / 2$ binsang geld.

36) tobu harean is suikerriet van een aanplant, waarvan de eigenaar nog niets gebruikt heeft; het snoepen van een aanplant, waarvan de eigenaar geregeld plukt, wordt blijkbaar niet als diefstal beschouwd.

$\left.{ }^{37}\right)$ Hoe Vg. aan zijn vertaling van tuak djantar komt weet ik niet; volgens vdT. is djantar een grote bamboekoker voor palmwijn, en wordt dus hier het stelen van reeds getapte en in een koker bewaarde palmwijn gesteld tegenover het stelen van sap uit de aan de boom hangende kleinere bamboekokers.

38) Boete voor een palmwijndief (si tangko pola) : D : 1 ampang zout.

39) lompisan ook weer: van een onaangebroken aanplant.

40) Voor manuk lahi bini vindt men meestal opgegeven: een witte en een rode haan.

41) Boete op het stelen van een bubu: D: 1 djual zout.

42) Een bubu staat met de opening stroomafwaarts, een tanggal met de opening stroomopwaarts.

43) Boete op het stelen van een tanggal: D: een parmasan zout. Dezelfde boete staat op het stelen van pisang ( $\mathrm{gaol}$ ). 
dihata radja, tamba ma utangna hataon ni radja di harungguan i.... [31, 16] Ia uhum ni halak sitangko sunut, dahanon sasolup, pira ni manuk $2 \ldots$.

[31, 17] Ia uhum ni halak na mamorus hamindjon, molo halak tandang, molo dapot sinoro, sipanganon ma i. Hape molo soada dapot, tu parmusuan ma i. Anggo halak di ladang ni iba do ibana, dapot pe sinoro, indadong i panganon; intap tu beangan do bahenon. Dung pe $i$ asa hinata utangna. Ia godang ni utangna: 3 binsang, 2 babi na bolon, sadampang dahanonna....

$[32,4])$ Ia uhum ni halak na mardjehe-djehe, molo dapot, sipanganon ma i. Hape molo na dung sanga laho tu huta ni halak, utangna ma sihataon. Ia godang ni utangna: 12 binsang, 1 horbo panulana, 12 sibalambung dahanonna. Hape molo soada targararsa barang dongan soada mangambati, djolma mangadop ma i, djolma mandingding pe djadi. Hape molo adong halak na maniop, tu halak pe mangadop dohot mandingding djadi do. Hape molo soada halak na maniop, tu parsingir i ma mangadop ${ }^{44}$ ). Ia na gadisonna pe, indadong be halak na mangorai, lomo ni rohana do di parsingiranna....

$[32,5]$ Ia uhum ni halak na manurbu ramba sampe na sasuhat boni bidangna,anggo inda duru ni pargadongan, marutang do i. Ia godang ni utangna: 10 binsang, 1 horbo, 10 sibalambung dahanonna. Molo soada gararna, djolma mangadop ma i, djolma mandingding pe djadi. Anggo gadison indadong djadi....

$262[32,6]$ Ia uhum ni halak na manurbu huta, dipambeangkon / ma nasida saluhutna. Dung pe i asa hinata utangna. Ia godang ni utangna : 12 binsang, 1 horbo panulana, 12 sibalambung dahanonna, i ma intop api goarna. Dung ni barang sadia ugasan ni halak na mosok abulonna ma i. Hape molo na soada gararna, diadop ma i. Hape molo na soada halak maniop, digadis ma i. Sude ma nasida sahuta i margadis na marutang $i$, tuhorna pe dibagi. I ma uhum ni halak na manurbu huta. Hape molo soada dapot na manurbu i, pintor laho tu huta na pulik, saleleng ni arina molo dapotsa sai na dibuat do i. I ma uhumna.

$[32,2]$ Ia uhum ni halak ná mamunu inana barang amana barang hahana, marsitingting ma nasida na saharungguan $i$, mangaronron tu huta ni na mamunu i. Dung ni diindahani ma na ro $i$, dibuat ma djuhut ganup bodari ganup manogot panganon ni na ro i. Dung ni marhata ma nasida. Dibuat na mamunu i ma babi na bolon panganon ni radja

44) Deze zin ontbreekt in de vertaling: Is er niemand, die de vordering wil overnemen, dan wordt hij pandeling bij de schuldeiser. (Het .,deze” in de volgende zin slaat dus op de schuldeiser). 
na ro, dilehon mas 2 binsang dongan ni djuhut i. Dibuat sada nari babi na bolon panganon ni isi ni huta, dilehon mas 2 binsang bagion ni isi ni huta, i ma daram banua goarna. Dung ni mulak be ma halak tu hutana ....

$[33,1]$ Ia uhum ni boru na mahilolong sian sinondukna, ditaruhon pardjolma i ma tu hula-hulana. Ditaruhon parboru i ma tu nampunasa djolma i. Dung ni mambuat djuhut ma pardjolma i panganon ni hulahulana i. Dung ni mulak parboru i. Dung ni, molo diulakkon muse mahilolong, laho ma nampunasa djolma i manullang babi tu huta ni parboru i. Dihata sintua ma utang ni parboru. Ia godangna: 2 binsang, 1 babi na bolon, sadampang ${ }^{45}$ ) dahanonna. Dung ni mulak ma pardjolma i. Dung ni molo diulakkon muse mahilolong, tu beangan ma i; 263 sahat martunas beanganna asa malua $\left.\ldots{ }^{46}\right)$.

$[33,2]$ Ia uhum ni halak na mahilolong lahi-lahi sian djolmana, ro ma parboru tu huta ni paranak i manullang babi. Dung ni dihata sintua ma utang ni na mahilolong: 2 binsang, 1 babi na bolon, 2 sibalambung dahanonna. $\mathrm{I}[\mathrm{a}]$ dung ma i didjalo parboru $\mathrm{i}$, asa dilehon ma sariar tu sibahen hata, i ma pago-pago, mambahen paso-paso ma disi : „Molo tung diulangkon muse mahilolong sian borungki, na tardege masna; atik mate pe boru soada martinading anak, indadong situngguonna tu ahu masna”, i ma ninna parboru. „Olo ma tutu”, boti ma ninna paranak. Dung ni mulak ma parboru i tu hutana, diparhadjimot nampunasa djolma ma djolmana....

$[33,3]$ Ia uhum ni halak na masihampian, na sada do ompuna, sada ulaon nasida ${ }^{47}$ ), ulos do 3 siboanonna dohot djuhut 1 bahen talangke sumangot. Dung dibuat djuhut $i$, dihatahon tu simangot ni ompuna na mate ${ }^{48}$ ) dohot tu begu ni pangoli na mate. Dung sun dihatahon, dilehon ma ulos i sada tu ama ni pangoli na mate $i$, sada tu inana, sada tu djolmana hinabiana i. I ma ruhut na mangabia na marsada ompu. Ia na dung pulik be do ompuna, sipanimbangi ma i. Molo sipanimbangi, barang sadia mas pangoli ni na mate $i$, dipaulak ma $i$

45) dampang bij $\mathrm{Vg}$. is natuurlijk een verschrijving voor ampang.

46) D: De schuld ingeval een vrouw uit afkeer van haar man naar haar vader teruggaat (uhum magigi) bedraagt 13 realen boven de bruidschat (panantum ni mas, lett. inwikkeling van het goud), 2 realen als kleed voor de schoonzoon, 1 voor zijn vader, 2 voor zijn moeder, 1 als herinneringsgeld voor de scheidsrechter (partola).

${ }^{47}$ ) sada ulaon nasida heeft Vg. overgeslagen; lett. „wier werk één is”. Ik weet ook niet precies wat er mee bedoeld is.

48) ompuna na mate, niet: de grootvaders van den overledene, maar: hun overleden grootvader, d.w.z. de gemeenschappelijke grootvader van de overleden en de tweede echtgenoot. 
saluhut; ia djuhut binuatna, barang sadia balgana, saí ma dipaulak. I ma uhum ni sipanimbangi. Hape molo nadong dape anggi ni pangoli na mate $i$, ro ma parboru $\mathrm{i}$ tumaruhon ulos pandasdas, dilehon ma $\mathrm{i}$ tu anggi ni na mate $i$, asa dihabia djolma ni hahana, barang anggina pe boti do. I ma uhum ni halak na masihabiaan.

[34] Ia uhum ni halak na marhopok ugasan mago, sampe dua hali diparunggu na agoan, las soada na paboahon. Dung ni dilehon ma dahanon parnungnung ganup ripe di hu/ta $i$. Dung dilehon na agoan $\mathrm{i}$ dahanon i, dipaima, las soada do na paboahon, djadi dialap ma datu pande marhopok. Dung ro di huta i, dibuat ma djuhut sada panganon ni datu i. Dung ni didokkon ma na mamulung. Dung ni dipulung ma nasa gota ni haju dohot gota ni sulu-sulu ${ }^{49}$ ), dibuat ma dohot tambor ni na mate dohot pangudjian ni batara guru ${ }^{50}$ ), dibuat ma damar dohot pandalo ni onggang dohot imbalo ${ }^{51}$ ). Dung ni diduda ma tu losung. Sun diduda asa disaor ma dohot gota i. Dung ni diengge ma dahanon 2 solup. Dung mengge, asa disaor ma tu pulung-pulungan i. Dung ni didokkon datu i ma mambuat bulu suraton na pungguron, homhom buhu ${ }^{52}$ ) ma dibahen. Dung ni disurat ma, disi ma disurathon tabas ni parhopohon 7 bindu. Dung ni didokkon ma na masimaremare tu balian dohot masianaknihaju. Dung ni dibuat suhut i ma sada djuhut. Sun masak djuhut i dohot indahan, dipio ma isi ni huta i saluhut, dipiohon ma dohot datu i. Dung ni mangan ma nasida. Sun mangan marhata ma nasida. Dihata ma utang ni panangko molo dapot: 2 binsang, 1 babi parbaribaan, sadampang dahanonna. Dung ni diparbaga upa ni datu: 2 riar, 1 djuhut. Dung ni disuhari radja ma $\mathrm{i}$ : „Beha ma tung soada dapot panangko, gurunami, beha ma i ?” boti ma didok tu datu i. „Olo, radjanami, molo na so isi ni huta on do na mambuat ugasan na mago $i$, malua ma $i$, halak ruar ma sitangko ugasan na mago i”, boti ma didok datu i. „Olo, gurunami, beha ma tung manuk ni datu dapot?” boti ma ninna radja i. „Olo, radjanami, molo tung manukku dapot, partolbok ma hadatuonhi, ahu ma sigarar harugian ni suhut” boti ma didok datu i. „Olo ba, beha ma tung manuk

49) sulu-sulu vlgs. vdT. niet jonge pisangspruitsels, maar $=$ sudu-sudu, euphorbia antiquorum; volgens De Clercq-Pulle (no. 1643) Euphorbia neriifolia L., een heester met giftig melksap.

$\left.{ }^{50}\right)$ n.1. tjèbok-bladeren.

51) imbalo, zwarte gom, ontbr. bij Vg. Het boomsap wordt voor deze gelegenheid door helpers van de datu verzameld (dipulung); de andere ingrediënten neemt hij (dibuat) uit zijn voorraad, stampt ze fijn in een rijstblok en vermengt ze vervolgens met het sap.

52) homhom buhu zal wel betekenen: een stuk aan beide zijden door een tussenschot gesloten. 
ni suhut dapot?" boti ma ninna radja i. ,Molo manuk ni suhut dapot, radjanami, tolbok ni suhut ma $i$, indadong na tutu mago ugasanna, mambuat / djuhut ma ibana panganon ni hamu isi ni huta, dibuat sada panganonhu, dilehon sariar hepeng di ahu; i ma uhumna molo manuk ni suhut dapot", boti ma didok datu i. Dung ni sun ma nasida na marhata i. Dung ni torang ma ari, dihandit ma losung, dipeakkon tu tonga ni alaman, dung ni dipagohon ma anak ni haju i, pinordalihan ma dibahen, dung ni dihaliangkon ma mare-mare $i^{53}$ ). Dung ni ditiptip ma bulu songon djangka ma dibahen ${ }^{54}$ ). Dung ni bongkot ma tu bogasan lobuan i, hundul ma tu atas losung i. Dung ni dilehon ma dahanon i sada tulpang didum naposo ni datu i. Dung ni dibasa datu i ma surat $i$, molo suda didum dahanon $i$, na bonar ma i. Dung ni marsiruhu ma ibana. Dung ni tole ma naposo ni suhut i. Dung ni tole ma isi ni huta i. Molo indadong tarbondutsa be dahanon na satulpang $i$, nungnga marbirsak-birsak sian babana, sahat sun dibasa datu i surat na 7 bindu i, indadong suda dibondut, i ma panangko $i$, na dung dapot ma i. Dung ni dibuat ma djuhut pardua-riaran, dilehon si 2 binsang i. Hape molo soada gararna, diadop ma i, tu parsingir i pe mangadop ibana djadi do. Dung ni dibuat suhut i ma djuhut sada panganon ni datu i, dilehon ma hepeng si 2 riar i. Dung ni mulak ma datu i.... Ia uhum ni halak na masirampasan tuan boru barang anggina dohot hahana, ro ma parboru i manopot tu huta ni pardjolma. Dung ni dibuat ma djuhut sada panganon ni parboru i. Dung ni marhata ma disi, dilehon nampunasa djolma i ma mas 2 binsang. Dung ni mulak ma parboru i.... ${ }^{55}$ ).

$[32,7]$ Ia uhum ni parpanguluon na tarose, na dung sun na hinatana,

53) $\mathrm{Bij}$ Vg. niet zeer duidelijk. De drie jonge boompjes (anak ni haju) worden in een driehoek om het rijstblok in de grond gestoken, en daaraan worden de tot franje uiteengehaalde jonge palmbladeren bevestigd, evenals in hoofdstuk 26 om de slachtpaal.

54) Door Vg. niet begrepen, daar in de door hem gebruikte transcriptie blijkbaar boeloeng i.p.v. boeloe stond. Er staat: Hij (de datu) snijdt een bamboetje glad af in de vorm van een (bij de Bataks van bamboe vervaardigde) patroonhuls. (Dit bamboekokertje moet zeker als maatje voor de te kauwen rijst dienen, en dus de inhoud van een pijpekop hebben).

55) Deze paragraaf ontbreekt bij Vg. De strekking is ook mij niet geheel duidelijk. Er staat: Indien mensen twisten om het bezit van een vrouw, bijv. twee broers, dan komt de vader van de vrouw naar het dorp van de eigenaar van de vrouw. Deze neemt een slachtbeest om de vader van de vrouw een maaltijd aan te bieden. Daarna bespreekt men de zaak; de eigenaar van de vrouw geeft 2 binsang goud. Dan keert de vader van de vrouw naar zijn dorp terug. (De schoonvader, of a.s. schoonvader, demonstreert blijkbaar door zijn bezoek, wie van de twistenden de rechthebbende is). 
djadi mangose na sambariba, marmusu ma pangulu $i$, dialo ma na mangose i. Dung ni marsada hasuhuton ma pangulu dohot suhut na $266 \mathrm{nio} / \mathrm{se}$ i....

$[32,10]$ Ia uhum ni halak na marbinda, morhuta-huta ma dibahen sada pangaradjai ; i ma partungguan ni nampunasa, i ma sidjalo garar ni parbinda. Dung ni dibahen padan barang sisabulan barang sidua bulan. Molo salpu padanna i inda digarar, binda bok ma gararon, na sahupang bindana djadi tonga riar digarar, na tonga riar bindana djadi sariar digarar; i ma uhum ni binda bok. Hape molo sanga tolu bulan salpu padan inda digarar, arga ni horbo ma sigararon ni pangaradjai, ibana martunggu tu pabinda. Molo pasuang argana, molo di horbo pasuang harihir ma goarna, molo di lombu pasuang djorat ma goarna, molo di binda babi pasuang palangka ma goarna, molo di binda biang pasuang ungur-ungur ma goarna, molo di binda manuk pasuang sunut ma goarna....

$[32,8]$ Ia uhum ni halak sisampe dangdang, na dung sun digarar parutang, ro pangulu dipogo. Molo sanga sian harungguan dibahen parsingir mangido, mambuat djuhut ma na mamogo i panganon ni parsingir i. Barang sadia pinogona i, dipaulak saluhutna....

$[32,14]$ Ia uhum ni halak na manunda pinahanna, barang horbo barang lombuna, dipangan eme ni halak, ditaruhon nampunasa hauma i ma parbubean ${ }^{56}$ ) tu nampunasa pinahan i. Dung ni dihata sintua ma i, dibahen ma utang ni nampunasa pinahan i 2 riar, 1 djuhut, sasibalambung dahanonna; i ma utangna. Molo soada olo manggarar parpinahan i, djadi tu parmusuan ma i....

$[32,16]$ Ia uhum ni halak na mangabul na mate di musu di hasuhutonna, di sun ni gora ma i digarar. Ia godang ni abul: molo radja 267 niabulna 3 binsang, 1 djuh/ut, sasibalambung dahanonna ....; ia abul ni anggi ni radja: 2 binsang, 1 djuhut, saparmasan dahanonna; abul ni namora pe na saí do; molo abul ni anggi ni namora: padua lombu ${ }^{57}$ ), 1 djuhut, 10 solup dahanonna....; ia abul ni parripe: sabinsang, 1 djuhut, 2 suhat dahanonna....; ia abul ni na so marripe, halak mandagang barang anak sorang: 2 riar, 1 djuhut, sasuhat dahanonna....; ia abul ni hatoban: sariar, 1 djuhut, 3 solup dahanonna....; ia abul ni datu: 2 binsang, sada djuhut, :saparmasan dahanonna; abul ni parsinabul pe na saí do; ia abul ni suhut dos do i dohot abul ni radja; ia abul ni ulubalang dos dohot abul ni anggi ni namora;

56) parbubean $=$ een rijstzak; Vg. vertaalt: de stoppels, blijkbaar naar van Bataks verkregen inlichtingen.

${ }^{57}$ ) padua lombu $=6$ riar, zie boven blz. 118 noot 26. 
ia abul ni pande musu dohot parhara musu dohot partahi musu: sabinsang be do abul ni, sada be djuhut, saparmasan be dahanonna ${ }^{58}$ ). I ma uhum ni abul ni halak na mate di musu silehonon ni suhut.

$[32,11]$ Ia uhum ni halak ma masitorpaan parsingir dohot parutang, tutu ninna parsingir, inda tutu ninna parutang, barang halak na masidjohaan, barang halak na masituhasan, ro ma sintua ditolai ma i. Molo na masitolonan, sintap dengke binungkusan do panolonanna barang garut na lamot ${ }^{59}$ ) barang taha nanggar, i ma uhumna. Hape indadong sada rohanasida disi, laho tu habonaran tarida, martampul manuk ma i. Dung ni ditangkup ma manuk sada, diarga na torop ma i sauang argana molo na torop manuhor, djadi 2 uang ma argana molo tu tampul manuk. Barang ise na talu, i ma sigarar i. Hape molo halak ruar sada, isi ni huta sada, na martampul manuk i, mangalehon pansar 268 alaman ma nasida tu radja sariar, tonga riar sian na talu, tonga ri/ar sian na monang. Dung ni ditiop nasida ma manuk i, ditodo be ma hira manukna. Molo siamunna di na sahalak dohot tanggurungna, siambirangna ma di na sahalak dohot indorana. Dung sun ditonai be, ditampul ma rungkung ni manuk i, dipasombu ma martalasa. Dung mate, ditundjang ma tano lambung peahan ni manuk i; sae-saena ma i. Molo nungnga inda humurtik be manuk $i$, dibuat na monang i ma sian peahanna i. Dung ni ditunggu ma singirna i, na sariar ${ }^{60}$ ) singirna djadi 2 riar ma i didjalo. Hape anggo barang aha do parsigulutanna dibahen na tampul manuk, na saí godangna, na saí do gararonna. I ma uhum ni halak na martampul manuk.

$[32,12]$ Ia uhum ni halak na mangarunsur, tung laho bungkas inda dipaboa tu radja dohot tu namora, molo dapot ditangkup nasida isi ni huta i na laho bungkas i, dibeangkon ma na sapanganan i. Dung pe i molo nadong gararna barang donganna na manggarar asa dihata utangna. Ia godang ni utangna: 10 binsang, 1 horbo, $12^{61}$ ) sibalambung dahanonna. Ia soada gararna, tu pargadisan ma i dohot tu paradopan, anggo panganon indadong djadi....

$[32,13]$ Ia uhum ni halak na umpongkal bulusanna, tung laho ibana

58) $\mathrm{Bij} \mathrm{Vg}$. ontbreekt: de vergoeding voor een pande musu, een parhara musu en een partahi musu is voor elk hunner een binsang, een slachtbeest en een parmasan rijst. (Zie over deze functies hoofdst. 13; Bijdr. 103 blz. 362 en 407).

59) een gladde slijpsteen.

60) Niet de reaal voor de kip, maar het bedrag van de schuld (als het om een geldschuld gaat) wordt verdubbeld; ging de twist niet over een geldschuld, dan vindt geen verdubbeling plaats.

61) In het hs. 12 , niet 10 . 
tu huta ni musuna, soada na marari radja ${ }^{62}$ ), molo adong tondongna di huta $\mathrm{i}$, indadong $\mathrm{i}$ tu beangan, utangna do sihataon. Ia godang ni utangna: 9 binsang, 1 horbo, 9 sibalambung dahanonna. Hape molo soada gararna mangadop ma $i$, las parripe nama dibahen di huta $i$. Hape molo soadadong tondongna mangambati, tu gadis ma i. Ia lahilahi na umbalga, lomo ni roha ni musuna ma disi; ia na panganonna 269 di rohana, dipangan ma i, molo na gadisonna di rohana, digadis ma... $[32,17]$ Ia uhum sidabuon ni hita pangulu tu parhataan ni halak, molo otik do mula ni parbadaan, sintap ni si 2 binsang do utang ni parsala, 1 djuhut parbaribaan, sadampang dahanonna; i do uhum ni. Hape molo balga mula ni parbadaan, olat ni si 12 binsang do utangna, sada horbo, 12 parmasan dahanonna; i do uhum ni ${ }^{63}$ ).

$[32,9]$ Ia uhum ni halak na manangkang di utangna, molo sanga sian muruk dibahen parsingir mangido, barang sanga siampahaek, gurgur ma utangna gararonna. Ia gurgur ni utangna, molo na 3 binsang utang i, djadi 5 ma gararonna, 1 djuhut parbinsangi, 5 parmasan dahanonna... $[32,15]$ Ia uhum ni halak na marbalok di tano na so djadi torbang masiulaan, masiingot ginuris ni ompuna. Molo torbang i diula tu tano ni na pulik, tu parbadaan ma i. Hape molo na marsigulut di parbalohan, tu harungguan ma i diboanhon, asa diuhumi radja na pulik. Ia uhum ni radja: manolon ma diuhumi. Hape molo manolon ma ninna, diuhumi ma balga ni panolonan. Molo hali lubang panolonan, sanga marmare-mare marhudjur pamunu ma $\mathrm{i}$, maroros-oros marpiso panamboli ${ }^{64}$ ); i ma uhumna. Barang ise panolon, i ma sibahen i. Ia godang ni hudjur dohot piso 2 riar; ia godang ni oros tonga riar. Dung ni laho ma nasida tu parbalohan i, dihali ma lubang, dibahen ma anak ni haju di bibir ni lubang i maropat suhi, songon pangkalang

62) marari radja vdT. wdb. blz. 20: het hoofd van een vijandelijke huta kennis geven, dat men zijn gebied om bijzondere redenen (als b.v. wegens een feest) betreden zal; om niet als vijand beschouwd te worden.

63) M.i. wordt hier niet gesproken over bemiddelaarsloon, maar nog eens als algemene regel gesteld, dat men, als bemiddelaar optredend, als maximum boete in kleine zaken mag opleggen 2 binsang enz., in grote zaken 12 binsang enz. (Er staat sidabuon, niet sidabuan).

B4) Vg.'s opvatting, dat de mare-mare een symbolische aanduiding van dodende speren en de oros-oros van een dodend mes zou zijn, kan ik niet delen. De mare-mare (jonge palmbladeren, tot franje uiteengerafeld) moeten in natura aanwezig zijn; de speer (waarmee men bijv. bij een feest een karbouw doodsteekt), de oros-oros (vlgs. vdT niet: fijngesneden bladeren, maar een ring van hoorn, waarmede men een sjerp vastknoopt, als men er een geld-fulp in heeft) en het slachtmes zijn oorspronkelijk ook wel onderdelen van het eedzweringstoestel geweest, maar worden door geld vervangen. Niet degene, die de eed oplegt, maar hij die door het afleggen van de eed zijn recht bekrachtigt, betaalt. 
ni tanoman ma dibahen ${ }^{65}$ ). Dung ni dihaliangkon ma mare-mare $\mathrm{i}$. Dung ni dipeakkon ma sitompion rohot santi-santi dohot napuran 270 tolum bulung $\left.{ }^{66}\right)$. Dung ni ditonai na pato/lonhon i ma paruarian $\mathrm{i}^{87}$ ). Dung ni manolon ma na mandjoha i. Dung sun manolon, mulak be ma nasida tu hutana. Mola sanga songon i balga ni paruarian, sibau indahan ma i, sibau napuran, indadong olo be i masidegean huta, pala padjumpa pe di dalan indadong olo be i masipanganan napuran. I ma uhum ni halak na masitolonan.

$[35,1]$ Ia uhum ni halak na mambahen djudji, asa didjudjur ma djolo ari na uli. Dung ni asa dibahen ma lobuan. Sun dibahen, asa didjudjur ma muse ari na uli. Dung djumpa asa dituhor nasida ulu porang i ma sada babi barang parriari barang partolu-hupangi, diboan dahanon saparmasan, diboan sitompion sahundulan, nitak sapinggan. Dung ni asa ditonggo ma saluhut radja ni nasida na saharungguan $i$ dohot pardjudji. Dung ro saluhutna asa diparmasak ma babi inon dohot indahan inon. Dung ni asa ditapol ma ria-ria dohot sanggul tu lambung pintu ni ruma-ruma horong. Dung ni asa ditibali ma sitompion i dohot nitak i. Sun masak indahan i dohot djuhut inon, ditibali ma i tu pinggan. Dung ni dihatahon ma tu boraspati ni tano, tu na martua sombaon, tu Boru Saniang Naga, tu Boru na Mora dohot tu'debata na tolu. Dung ni dihatahon ma cu simangot ni pardjudji na djolo dohot tu Budjang Djuaro ${ }^{68}$ ). Dung sun dihatahon, dihehei nasida ma tinibalhonna i. Dung ni mangan ma nasida na di lobuan $i$ saluhutna. Sun mangan $\mid$ marhata ma nasida. Ro ma radja tinonggona i, disungkun ma saksi ni djudji : „Boti ma i, amang hamu ulu porang $i$, beha saksi ni djudjimuna on, asa binoto", boti ma didok/

271 radja na di pardudjian. ,Olo, radjanami, anggo saksi ni djudji on: $\mathrm{Na}$ so djadi marbada di bogasan on, anggo di balian i djadi do; molo marbada di bogasan on, sitompas robu ma $\mathrm{i}$; barang sadia harugiannami ${ }^{69}$ ) na mambahen pardjudjian on, na saí ma paulahonna; i ma saksi ni bada. Ia na djuara masisingiran, molo inda djolo disungkun hami ulu porang, na so djadi masiihotan. Anggo na masitungguan,

65) Vert.: boomstammetjes worden langs de rand van de kuil in een rechthoek gelegd, als de rand van een graf.

66) De kippeneieren staan niet in de tekst, wel offerrijst (santi-santi).

${ }^{67}$ ) Vert.: De eedoplegger bezweert het eedzweringsapparaat (vgl. Vg. blz. 144 noot 2).

68) Budjang Djuaro is een eigennaam, blijkbaar van een beschermgeest van het dobbelspel, en blijkens de vorm djuaro i.p.v. djuara aan het Minangkabaus ontleend. Vg. vertaalt na djuara steeds door bankhouders; vdT. geeft: de spelenden op een dobbelpartij, hetgeen in deze tekst beter uitkomt.

69) Niet schade, maar kosten. 
djadi do. Molo tung diihoti parsingiranna, inda disungkun hami, marutang ma i. Ia godang ni utangna: sada riar, sada djuhut, sasuhat dahanonna; i ma utangna. Ia di saksi ni gade, sian ulu binahen tu ulu, sian gonting binahen tu gonting, sian pamatang binahen tu pamatang, sian hombal binahen tu hombal. Ia na mago gade, mago utangna; ia na maribak gade, mago anak ni hepeng. Hape molo na dituntun diagohon dohot diribakkon, arga ni ugasan sigararon ni sitiop gade. Ia dipadanna, pitu borngin inda [ditobus] di djudji inon, pate gadena; molo di mate ni djudji, dua hali pitu borngin dung mate djudji inda ditobus gadena, pate ma i. Ba ia di hepeng tanggungan ${ }^{70}$ ), sampe pitu borngin inda digarar, lohot tanggunganna. Ia godang ni tanggungan: na satali djadi sariar gararonna. Ia ugasan tinanggungna mararga sahupang, ugasan 2 riar gararonna molo mago ugasan tinanggungna i; ba i ma uhum ni. Ia di tuhuhan, na soada marimpit be paraloanna, ianggo uhum ni hami ulu porang si sahupang do utang tuhuhan, salpu i indadong utang be $i$, molo indang disungkun pardjudji hami ulu porang. Hape molo huorai hami ulu porang, sai didatdati nasida, lomo ni rohana be, inda di hami be, uhum / ni nasida be nama disi, i ma uhum ni tuhuhan. Ia saksi ni halak na marutang, ianggo si tonga riar do utang ni halak, indadong ${ }^{71}$ ) djadi ihotan. Hape molo sampe sariar, sungkunon ma taonna. Hape molo na'soada taonna, gadena pinangido. Hape molo soada gadena, niompashon tu tondongna. Hape molo soada olo tondongna manggarar utangna i, dung pe $i$ asa bineangkon. Molo nungnga sanga tu beangan, molo adong tondongna na mangambati, gurgur ma utang gararonna: dilehon ma harhar tali tonga riar, tangtang beangan sariar, singkat ni na pinangan sariar, djuhut 1 , dahanon sadjual; i ma uhum ni. Hape-molo soada tondongna mangambati, gadison ma i. Anggo utang ni pardjudji, atik songon dia pe godang ni utangna, molo indadong didaishon tu pardihutana, na so djadi do $\mathrm{i}$ tungguon tu inana barang tu djolmana barang tu amana; i ma uhum ni. Ia tuhum ni dadu, sipat tabung do daona sian pandabuan. Ia na mago dadu dibahen na djuara, molo dituntun disampathon do dibahen na mago, sariar sada-argana. Hape anggo na dipastap do dohot tangan tonga riar sada argana. Ia na mabola dadu dibahen na djuara, ianggo tanganna do dibahen, barang

70) Het door vdT. wdb. blz. 223 onder tanggung I gegevene: ,geldbedrag dat men tegen een bepaalden tijd, zo het uitgeleende te zoek is geraakt, van den leener vordert" slaat wel op deze plaats. satali schijnt hier $1 / 2$ hupang te zijn, dus 40 duiten, wat echter niet klopt met de opgaaf van vdT. wdb. blz. 375: satali $=24$ duiten.

71) Het woord „niet" is in Vg.'s vertaling uitgevallen.

D1. 105. 
tabung dilotakkon, indadong sigararonna i. Alai molo tung hepeng dilotakkon, barang haju barang batu, gararonna ma i, sariar sada ma argana; i ma uhum ni ${ }^{72}$ ). Ia di hepeng margantung tu gadena, molo martobus ibana, djolo hepeng na margantung do dilehon asa tiniop ni gadena; alai molo nungnga dilehon na margantung, na so 273 djadi pate be gadena; i ma $\cdot$ uhum ni ${ }^{73}$ ). Ia u/hum ni na manuruni, na so djadi do i; molo adong hepengna didjadihon; molo nadong gadena, hoelehon hami hepeng; anggo tung manuruni, na so djadi do i. Molo tu isi ni hutaniba, ba i ma uhumniba. Ia uhum ni halak na mambahen djudji di balik ni djudjinami on, nasa na dung dohot mangan pamosik on, molo tung dipungka djudjina di na pulik inda disungkun di hami, silangkup djudji ma i, marutang ma i bahenon. Ia godang ni utangna: 1 babi na bolon, sabinsang batuna, sadampang dahanonna. I ma uhum ni djudjinami on, radjanami”, boti ma didok ulu porang i. „Olo ba, molo i ma uhum ni djudjimuna i, djadi do antong”, boti ma didok radja na di pardjudjian i. Dung ni dipahembang ulu porang i ma lapik tu parbandaan ni pardjudjian i. Dung ni dipadalan ma hepeng tu pardjudji i, dilehon ma satali be; i ma tompas porang goarna, indadong na margade $i$, maranak pe indadong. Dung ni mardjudji ma halak. I ma uhum ni na mambahen pardjudjian dohot saksi ni pardjudjian.

Ia uhum ni paraloan di na godang dohot di na otik ${ }^{74}$ ). Molo gereran,

$\left.{ }^{72}\right)$ Deze passage ontbreekt in de vertaling. Vert.: Maakt de speler een dobbelsteen stuk (lett. gespleten), dan behoeft hij die niet te betalen indien hij het met zijn hand doet of als hij er met de werpkoker op slaat. Slaat hij er echter met eén geldstuk, met een stuk hout of met een steen op, dan moet hij een reaal per stuk betalen.

73) Onduidelijk bij Vg. Vert.: Wat betreft geld geleend boven de pandsom, bij inlossing moet men eerst dit extra geleende terugbetalen, dan pas de eigenlijke pandsom. Als het extra geleende terugbetaald is, kan het pand niet meer aan de houder vervallen.

$\left.{ }^{74}\right)$ Het hier volgende gedeelte is in Vg.'s vertaling overgeslagen. De technische termen zijn zonder verklaring van een deskundige niet te begrijpen; het woordenboek van vdT. schiet hier tekort. Het hier beschreven dobbelspel komt niet overeen met het Batakse dobbelen, dat ik een paar maal nog in Simaloengoen en de Karolanden zag. Uit de beschrijving maak ik het volgende op: Het spel wordt gespeeld door twee spelers, van wie één, pandabu, de dobbelstenen werpt; de ander heet tahanan dadu. Welke soort en hoeveel dobbelstenen (twee?) gebruikt worden, is niet duidelijk. De inzet (paraloan) van beide spelers is gelijk. Er zijn vele worpen mogelijk; sommige betekenen gelijk spel (sari, uit het Minangkabaus $=$ Maleis seri); verder heeft men torus, waarbij de totale inzet aan de winnaar,komt; mandangka (vdT.: twee opéénvolgende getallen opwerpen van dobbelstenen), waarbij meestal de winnaar zijn eigen inzet plus een gedeelte van de inzet van de verliezer krijgt; en nog een vierde mogelijkheid, genoemd singirna. Eerst worden de namen van de verschillende inzetten 
sauang etek ma i, indadong mandangka be i. Molo unsung mirik, 3 hepeng ma i sian pandabu, 3 sian tahanan dadu; molo mandangka do dadu i, sahepeng do hamonanganna, 2 hepeng mulak tu na talu. Molo 2 uluna 4 ihurna, 6 hepeng be ma paraloan nasida; m.m.d.d.i., 2 hepeng do h. sauang etek m.t.n.t.; hape molo torus dadu i, saluhutna ma i di na monang. Molo torus sauang ninna, sauang be ma nasida; m.m.d.d.i, sauang etek do h., sauang etek m.t.n.t. Molo sauang dua 274 etek paraloan, tonga dua uang be ma / dibahen nasida; m.m.d.d.i, sauang etek do h., sauang m.t.n.t.; alai molo torus, di na monang ma saluhutna. Molo sauang suang goarna, 2 uang be ma nasida ; m.m.d.d.i, sauang do h., sauang m.t.n.t.; molo torus dadu i, di na monang ma saluhutna. Ia sauang dua goarna, 3 uang be ma paraloan nasida; m.m.d.d.i, sauang do di na monang, 2 uang m.t.n.t. Molo dua-dua uang goarna, opat uang be ma paraloan nasida; m.m.d.d.i, 2 uang h., 2 uang m.t.n.t. Ia tali-tali goarna, 6 uang be ma paraloan nasida; m.m.d.d.i, $\dot{3}$ uang ma i di na monang, 3 uang m.t.n.t.; ia torus do dadu i, saluhut ma i di na monang; i ma uhum ni. Ia 4 ihurna' 3 uluna, 7 uang be ma paraloan nasida; m.m.d.d.i, 3 uang do h., 4 uang m.t.n.t.; i ma uhum ni. Molo 2 hali 4 uang, 8 uang be ma paraloan nasida; m.m.d.d.i, 4 uang do h., 4 uang m.t.n.t.; ia torus do dadu i, saluhut ma i di na monang; i ma uhum ni. Ia suhu-suhu uang goarna, 12 uang be nama nasida; m.m.d.d.i, 6 uang ma di na monang, 6 uang m.t.n.t.; molo torus do dadu i, di na monang ma i saluhutna; $i$ ma uhum ni. Ia suhu-suhu lapik goarna, 16 uang be ma paraloan nasida; m.m.d.d.i, 6 uang do h., tonga riar m.t.n.t.; ia torus do /dadu i, di na monang 275 ma saluhutna; $i$ ma uhum ni. Atik suhu-suhu riar pe goar/na, ianggo bilanganna dohot parbagina songon i do. $\{$ Ia paro-paro uang goarna, sariar 4 uang be ma nasida; m.m.d.d.i, tonga riar ma di na monang, 14 uang m.t.n.t.; hape molo torus dadu i, d.n.m.m.s. ; i ma uhum ni. Molo paro-paro lapik goarna, 6 hupang ma i; m.m.d.d.i, tonga riar

genoemd, te beginnen bij gereran (de kleinste, elk 4 duiten) tot de grootste, paro-paro lapik of paro-paro riar geheten (elk $11 / 2$ reaal), met de verdeling van de inzet in het geval van mandangka. Dan worden enkele bizondere speelwijzen verklaard, bijv. martamuse, waarbij men de inzet niet telt, doch een willekeurige hoop geld neersmijt; in geval van torus krijgt de winnaar alles, anders gaat men tellen en wordt gelijk op gedeeld. Daarna wordt de betekenis van sari verklaard en enkele gevallen van singir genoemd. Vallen de dobbelstenen buiten de mat, dan geldt het niet. Als de oeloe porang bezig is de worp te beoordelen, en een derde komt en de dobbelstenen beweegt, dan wordt de beweger beboet. De inzet komt aan de winnaar; een bedrag gelijk aan de inzet moet door de beweger aan de verliezer betaald worden. Hierna volgt het tweede gedeelte van Vg.'s hoofdstuk 35 . 
do hamonanganna, sariar m.t.n.t.; hape molo torus dadu ' $i$, saluhut ma di na monang; i ma uhum ni. Atik paro-paro riar pe ninna goar, songon i do parbagina; i ma uhum ni paraloan. Molo bolangkang ninna goarna, m.m.d.d.i, mulak be ma paraloanna $i$; hape molo torus do dadu i, d.n.m.m.s.; i ma uhum ni na marbolangkang. Ia marsantuhuk goarna, molo sari do na sahali mandabu i dadu i. mulak be paraloan; alai molo singirna barang mandangka pe i barang torus pe dadu i, sai di na monang do saluhutna paraloan i.... Molo tamuse ninna goarna, indadong dibilang be hepeng, ba rung nama ${ }^{75}$ ) sada, ditinggangkon pangimpitna; ;torus ni dadu nama guruna; molo torus dadu i, dibuat na monang i ma saluhutna; hape molo indadong torus, dibilang ma i: sitonga di na monang, sitonga mulak tu na talu.... Ia marlamo-lamo goarna, j18 uang be paraloan nasida; m.m.d.d.i, 8 uang tu na talu mulak, tonga riar tu na monang; hape molo torus dadu i, d.n.m.m.s.... Ia uhum ni dadu na sari, molo onom-onom na sada, barang lima-lima satu-satu na sada, barang dua-dua na sada, barang 276 djongdjong na sada / peak na sada, na sari do i; molo na djeng dadu, i ma siuhumon ni ulu porang. Hape molo tung djongdjo[ng] dadu i saluhutna, sitoro ${ }^{76}$ ) i dompak gindjang, singir ni pandabu ma i. Hape molo sitambon ni bodat tu gindjang, singir ni tahanan dadu ma i. Hape molo haruar lapik dadu i, na so djudji do i. Molo manguhum ulu porang di dadu, tung ro na pulik, tung humurtik dibahen dadu , i, marutang ma $i$; ia paraloan dibuat na monang ma $i$; barang sadia paraloan $i$ gararon ni na mangkurtik i ma tu na talu; i ma uhum ni.

[35, 2] Ia dung ma leleng halak mardjudji, molo adong halak na tu tali barang na tu beangan, diompashon ulu porang ma tu donganna barang tu hula-hulana barang tu ianakkonna. Ingkon na so olo be mangambati asa djadi gadison halak na tu beangan i; i ma uhum ni. Ia uhum ni na dung mate djudji, asa diduda ulu porang i ma dahanon bahen nitak, dituhor pola. Dung ni didjou ma saluhut nasa na mangan pamosik i dohot pardjudji saluhut. Dung ni asa dilehon ma nitak i dipangan halak dohot pola i diinum halak. Dung sun minum asa manungkun ma radja na di lobuan i: „Boti ma i amang hamuna ulu porang $i$, aha ma nidokmu dibahen dilehon hamu nitak panganonnami dohot pola inumonnami ?” boti ma didok radja i. ,Olo radjanami,

75) Of barungna ma? (barung dan in de door Warneck opgegeven betekenis van „duit”, bij vdT. baru). De komma na ditinggangkon moet dan vervallen.

76) De tweede lettergreep van dit mij onbekende woord is onduidelijk in het hs. 
hubahen hami pe i, paboa na dung mate do djudji, asa diboto hamu radja dohot namora dohot na djuara. Ia tung so hupaboa hami, tung songon nạ muningan hamu radja dohot namora dohot na djuara, in/ 277 [da] diboto tobusonna gadena. On pe radjanami, i do nidok ni. Sintap 2 hali 7 borngin on masitobus gadena ma djuara. Hira ompas-ompasnami ma on, molo so ditobus gadena, salpu si 2 hali 7 borngin on, pate ma gadena”, i ma didok ulu porang i. „Olo amang, molo i ma hape nidokmuna, paboa djudji ma hape na dung mate, asa diboto na djuara manobus gadena, djadi tutu. Ale amang hamuna na djuara i, bege hamu hata ni ulu porang i, salpu 2 hali 7 borngin on masitobus gadena, pate ma i", boti ma didok radja i. Dung sun nasida mangkatai mulak be ma nasida. Dung ni nasa nadong bahenonna ditobus ma gadena, molo soada bahenonna pate ma gadena. I ma uhum ni halak na mate djudji.

[36] Ia uhum ni halak na mambahen onan, asa marrunggu ma nasida na saharungguan i saluhutna. Dung ni asa dirabi nasida ma sibahenon onan i. Dung sun dirabi nasida, asa didokkon ma na mangalapi tu huta na pulik ${ }^{77}$ ). Dung ni asa ro ma nialapna $i$, asa marpungu ma halak di onan i, masiboan djagalna ${ }^{78}$ ) ma halak. Dung ni asa dilehon nasida parbona ni onan i ma tuak tu na nialapna i. Dung sun halak minum, manungkun ma radja na ro $\mathrm{i}$ : „Boti ma i, hamu parbona ni onan on, aha ma nidokmu dibahen na minum pola hami dibahen hamu?" boti ma didok radja nialapna i. „Olo radjanami, hulehon (hon) hami pe i diinum hamuna, paboa na dung adong do onan hubahen hami dison, asa diboto hamuna do marboan boniaga tu onan on antong; ianggo panggurguri ni onanta on, djumpa a/ri na uli disi ma itabahen”, boti ma ninna parbona ni onan i. „Olo ba tutu, molo i ma hape, djadi do i antong", boti ma didok radja na tinonggona i. Dung ni dibahen ma padan haroro ni radja tinonggona i. Dung ni sun ma nasida na marhata i. Dung ni mulak be ma halak tu hutana. Dung ni margugu ma nasida parbona ni onan i manuhor horbo sada. Dung ni digugu nasida ma dahanon, dung pungu guguan nasida $i$, disuhat ma dahanon i sampe 7 ampang godangna. Dung djumpa ma padan i, marhobas be ma halak sian hutana laho tu onan i. Dung ni ro ma nasida parbona ni onan $i$, dibahen ma sitompion sahundulan, nitak sapinggan. Dung ni borhat ma nasida, dohot ma boru-boruna. Dung ni ditogu ma horbo i, dilompai ma dohot indahan, dipapulik ma pusu-pusu i sambola bahen parbulanan ni nasida parbona ni onan

77) In de door Vg. gebruikte transcriptie stond blijkbaar pitu i.p.v. pulik.

78) djagal vdT.: koopwaar, vooral die in eetwaren bestaat. (Vg.: vlees). 
i. Dung ro halak saluhutna, mangan ma nasida. Marhuta-huta ma dibahen tanggo-tanggo ni horbo i. Dung ni asa dibagi ma indahan tumpa-tumpa $\left.{ }^{79}\right)$, marhuta-huta ma dibahen. Dung sun halak mangan, asa marpungu ma nasa sintua, marpungu ma nasida suhut $i$ saluhutna. Dung ni ditiop nasida ma pusu-pusu, marsahuta-sahuta sahalak mandok. Dung ni ditonai ma pusu-pusu i : ,Ale ompung pusu-pusu ginantung, na saut mate na sundat mangolu ${ }^{80}$ ), molo tung hutinggalhon donganhu parbona ni onan, atik adong halak na manompas saksi,

279 inda sai rap mangalo do hami, / digantung begu digantung debata na pinompar, na ginabe, na sinamot, na niula ${ }^{81}$ ); alai anggo rap do mangalo halak, horas hami”, boti ma ninna. Ganup ma nasida parbona ni onan i mandok i. Dung sun nasida na marbulan i, marhata ma nasida dohot radja tinonggona $i$, „Boti ma $i$, amang hamuna radja na tinonggo, ianggo sipanganon indada pala sauthononhon hamu; ba ia saksi ni onan sungkun hamu di hami asa hupaboa hami", boti ma didok nasida bona ni onan i. Dung ni disungkun na ro i ma saksi : „Boti ma i amang hamu bona ni onan on, ia panggurguri ni onan on nungnga hupangan hami, ia saksi ni onan paboa hamu asa huboto hami”, boti ma didok radja tinonggona i. „Olo amang, ianggo saksi ni onan on, na so djadi masibunuan, na so djadi martindju. Tung adong pe utang, anggo utang sian na pulik do mula ni utang ditunggu di onan on, tung soada pe garar ni parutang, na so djadi do masiihotan di onan on; alai anggo dison do mula ni utang, dison ditunggu, molo na soada gararna, na soada gadena, na soada taonna, djadi do diihoti; ba i [ma] uhum ni. Ia di halak na manompas saksi, tung dibunu halak di onan on, barang laho mulak, molo di tano na hugomgom hami do dibuat halak, marlulu do hami; didjudjur tinading ni na tardobo lungun ni rohana, hululuhon hami saksinami. Molo na marnihot halak di onan on, inda tandap huboto hami, marutang ma i; barang sadia pang280 gurguri ni onan on hubahen hami, na saí ma paulahon/na. Hape anggo na laho tu onan on tung dibuat halak di dalan, pangaluhon ni tinading ni na tardobo do tu hami. Hape na marbada halak di onan on, molo sanga dabu tangan be nasida, marutang ma i 1 djuhut, sariar hepeng, sadjual dahanonna; i ma utangna. Ia na sanga tiris mudar halak, umbalga ma utang ni na mambahen i: 2 binsang hepeng, 1 babi

${ }^{79}$ ) indahan tumpa-tumpa, vdT.: gekookte rijst in buideltjes, die men naast de bestemde schotels plaatst, om de gasten zo zii soms niet genoeg mochten hebben, de gelegenheid te geven zich zelve te bedienen.

80) Vert. : dat definitief dood is, dat beslist niet meer leeft.

81) Vert.: dan mogen de geesten ophangen en de goden opschorten mijn nakroost, mijn zegeningen, mijn voorspoed, de vruchten van mijn arbeid. 
na bolon, 2 sibalambung dahanonna; i ma uhum ni. Ia boru-boru marbada dohot lahi-lahi, molo sanga lalu tanganna tu boru-boru, barang marsae-mara dibahen, (marutang) marutang ma i sabinsang mas, 1 babi parbaribaan, saparmasan dahanonna; i ma uhum ni. Ia uhum ni halak na mamolahon solup di onan on, marutang ma i 1 babi na bolon, 3 binsang batuna, 3 parmasan dahanonna; i ma uhum ni. Ia uhum ni halak na manangko sjan onan on, molo napuran ditangko barang dahanon barang aha djagal metmet, molo tarboto, utangna do sihataon. Ia godang ni utangna: 1 riar, 1 djuhut, sadjual dahanonna; i ma utangna. Hape molo hepeng ditangko barang mas barang ulos, sipanganon ma i, molo soada tondongna mangambati. Hape molo adong tondongna, utangna ma sihataon. Ia godang ni utangna: 5 binsang, 1 horbo, 3 ampang dahanonna; i ma uhum ni”, boti ma didok parbona ni onan i. „Olo amang, molo i ma saksi ni onan on, djadi ma tutu", boti ma didok na tinonggo i. Dung sun ma nasida na marhata i, laho be ma halak mulak. Dung djumpa 4 borngin, ro ma halak maronan. I ma uhum ni halak na mambahen onan, 4 borngin tingkina ${ }^{82}$ ).

s2) Het hoofdstuk over de marktstichting komt niet voor in het afschrift in Cod. 3416. In plaats daarvan vindt men daar het stuk over hetzelfde onderwerp uit $\mathrm{C}$. 